\title{
Mid-Latitude Ice on Mars: A Science Target for Planetary Climate Histories and an Exploration Target for In Situ Resources
}

\author{
A White Paper submitted to the Planetary Sciences Decadal Survey 2023-2032 \\ Primary Contact: \\ Ali M. Bramson, Purdue University (BramsonA@purdue.edu)
}

\begin{abstract}
Ali M. Bramson ${ }^{1,2}$
Chimira Andres ${ }^{3}$

Jonathan Bapst ${ }^{4}$

Patricio Becerra ${ }^{5}$

Samuel W. Courville 6

Colin M. Dundas ${ }^{7}$

Shannon M. Hibbard ${ }^{3}$

\author{
Authors: \\ John (Jack) W. Holt ${ }^{2}$ \\ Suniti Karunatillake ${ }^{8}$ \\ Aditya Khuller ${ }^{9}$ \\ Michael T. Mellon ${ }^{10}$ \\ Gareth A. Morgan ${ }^{6}$ \\ Rachel W. Obbard ${ }^{11}$ \\ Matthew R. Perry ${ }^{6}$
}

Eric I. Petersen ${ }^{2,12}$

Nathaniel E. Putzig ${ }^{6}$

Hanna G. Sizemore ${ }^{6}$

Isaac B. Smith ${ }^{6,13}$

David E. Stillman ${ }^{14}$

Paul Wooster ${ }^{15}$

${ }^{1}$ Purdue University, ${ }^{2}$ University of Arizona, ${ }^{3}$ University of Western Ontario CA, ${ }^{4} J e t$ Propulsion Laboratory, California Institute of Technology, ${ }^{5}$ University of Bern $\mathrm{CH}$, ${ }^{6}$ Planetary Science Institute, ${ }^{7}$ U.S. Geological Survey, ${ }^{8}$ Louisiana State University, ${ }^{9}$ Arizona State University, ${ }^{10}$ Cornell University, ${ }^{11}$ SETI Institute,

${ }^{12}$ University of Alaska Fairbanks, ${ }^{13}$ York University, ${ }^{14}$ Southwest Research Institute, ${ }^{15}$ SpaceX
\end{abstract}

Ken Herkenhoff, U.S. Geological Survey David M. Hollibaugh Baker, NASA GSFC Stefano Nerozzi, U. of Arizona Serina Diniega, NASA JPL Jeffrey Plaut, NASA JPL/Caltech Charity Phillips-Lander, SwRI Cynthia Dinwiddie, SwRI Aymeric Spiga, LMD/Sorbonne Université, FR Andreas Johnsson, U. of Gothenburg, SE Michael Mischna, NASA JPL/Caltech Adrian J. Brown, Plancius Research, LLC Noora Alsaeed, CU Boulder, LASP Rachael Hoover, SwRI Eleni Ravanis, European Space Agency Jorge Núñez, JHU/APL

Alice Lucchetti, INAF-OAPD Padova, IT Maurizio Pajola, INAF-OAPD Padova, IT

\section{Signatories:}

Alfred McEwen, U. of Arizona

Wendy Calvin, U. of Nevada Reno Nicolas Thomas, U. of Bern, $\mathrm{CH}$ Paul Hayne, U. of Colorado Boulder Kim Seelos, JHU/APL Shane Byrne, U. of Arizona Devanshu Jha, MVJ College of Eng., IN Michael S. Veto, Ball Aerospace Matthew Chojnacki, PSI Jacob Widmer, Representing Self Carol Stoker, NASA Ames Alberto G. Fairén, CSIC-INTA, ES Edgard G. Rivera-Valentín, LPI/USRA Marcella Roth, Lockheed Martin Philippe Sarrazin, SETI Corbin Kling, North Carolina State University 


\section{Summary}

In the last decade, aided by the high-resolution data and long-term monitoring by NASA's Mars Reconnaissance Orbiter (MRO) and other spacecraft, extensive evidence has emerged supporting the presence of abundant $\mathrm{H}_{2} \mathrm{O}$ ground ice throughout much of the mid-latitudes of Mars. Growing evidence indicates that much of this ice is relatively pure, exists within a few meters of the surface, and reaches lower latitudes than previously thought, potentially providing an accessible record of the recent climate and a large in situ resource for future human exploration of Mars. We are reaching the limits of currently available datasets, however, just as we are starting to unlock the climate record and determine the water resources contained within the Martian mid-latitudes. A comprehensive understanding of the nature of this ice would significantly enhance our understanding of Mars' climate history and total water budget, as well as the effects of orbital/axial forcing on volatiles. In this regard, Mars is a testbed for comparative planetary climate studies, including for exoplanets; these studies are particularly valuable because Mars has many similarities to Earth but lacks the complicating effects of oceans and a biosphere, such that orbital/axial forcing dominates climate variability.

Quantifying the volumes, distribution, and properties of the water ice are crucial for addressing two overarching questions in the next decade:

1. What climate record is preserved in mid-latitude ice deposits on Mars?

2. How accessible is the ice as a resource for future exploration?

New missions will enable us to capitalize on the major discoveries of the last decade and take the next giant leap in the upcoming decade to address these questions.

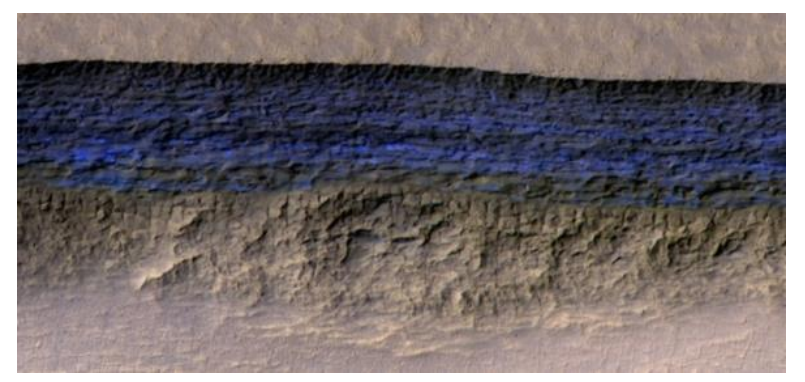

Figure 1. Site where a scarp exposes a thick ( 100 m) $\mathrm{H}_{2} \mathrm{O}$ ice-rich deposit in the subsurface (Dundas et al., 2018), MRO HiRISE image ESP_022389_1230.

\section{Background}

\section{Orbital and Climatic Control of Subsurface Water Ice}

Perturbations of a planet's orbital parameters (e.g., eccentricity and longitude of perihelion), and tilt of its rotational axes (i.e., obliquity) play an important role in the climate history of that planet. While Mars' current obliquity $\left(25.2^{\circ}\right)$ is similar to Earth's $\left(\sim 24^{\circ}\right)$, it has undergone extremely large variations over the course of the planet's history, from as low as $0^{\circ}$ to likely higher than $60^{\circ}$ (e.g., Laskar et al., 2004). These perturbations influence the distribution of solar insolation across the planet and are thought to be the main driver of many atmospheric and surface processes that have occurred during much of the Amazonian period (last 3 Gyr) (e.g., Toon et al., 1980; Jakosky, 1985).

In the present climate, subsurface ice is stable in parts of the Martian mid-latitudes, with a distribution that depends on the temperature regime, depositional history in regards to lithic content and overburden, and atmospheric water vapor content. Adjustments to the depth to the ice (i.e., the ice table) are expected to be geologically rapid, but thick ice 
units can survive while ice is lost, especially in the presence of an insulating surface layer of dust. The global distribution of ice is not well known, since most current remote sensing data sets are either only sensitive to the shallowest ice (on the order of tens of centimeters) or require a thick, pure unit (more than tens of meters). Determining the ice's distribution in detail and establishing its current stability state will inform us about the integrated history of ice accumulation and volatile transport between different areas over geologically recent time.

\section{Observations of Martian Subsurface Ice}

At present-day, water ice is only perennially stable on the surface at the poles, with ice being stable at lower latitudes in the subsurface. Due to the inverse relationship between temperature and latitude, ice must be buried at greater depth for increasingly equatorward locations (e.g., Leighton and Murray, 1966). The Phoenix lander excavated ice in the upper centimeters of the surface at $68^{\circ} \mathrm{N}$ (e.g., Smith et al., 2009), while recent (formed in the last 15 years) impact craters have exposed and excavated nearly pure water ice (likely $>90 \%$ ice by volume) within a meter of the surface as close to the equator as $39^{\circ} \mathrm{N}$ (Byrne et al., 2009; Dundas et al., 2014). Indeed, water equivalent hydrogen maps based on gamma ray and neutron spectroscopy show evidence for widespread ice in the mid-to-high latitudes within $\sim 1 \mathrm{~m}$ of the surface (e.g., Pathare et al., 2018; Wilson et al., 2018). An ice-rich latitude-dependent mantle (LDM) ranges from a few to tens of meters in thickness throughout both the north and south mid-latitudes (Squyres and Carr, 1986). Glacial features are abundant, and include lineated valley fill (LVF) and concentric crater fill (CCF) (e.g., Levy et al., 2014). Through a combination of geomorphic and radar analysis, lobate debris aprons (LDAs) are now known to be remnant debris-covered glaciers of nearly pure ice (e.g., Holt et al., 2008; Plaut et al., 2009). Additionally, radar sounding has detected subsurface interfaces attributed to the bottom of massive ice sheets buried across the northern plains regions of Arcadia Planitia (Bramson et al., 2015) and Utopia Planitia (Stuurman et al., 2016), though evidence is conflicting regarding the bulk purity and abundance of ice in these units (Campbell and Morgan, 2018). Scarps near $\sim 55^{\circ}$ latitude (in both the north and south) expose thick, massive ice (Figure 1) and appear to extend to very near the surface in high resolution images (Dundas et al., 2018), providing the most concrete evidence that the deep radar signatures of ice may be the base of a thick, continuous reservoir that extends to the shallowest detections of ice. Ultimately, the structure of the near subsurface (upper tens of meters), including the depth to the ice table and amount of lateral and vertical heterogeneities, is not well understood, especially at lower latitudes where the ice is likely less stable in the present obliquity.

The ice concentration and its vertical structure are critical tracers of deposition and modification processes and the variability in these processes over time. Ice deposited from vapor will primarily fill in regolith pore space as "pore-filling ice," with the resulting ice concentrations being equal to (or occasionally less than) the available pore space. Meanwhile, snow accumulation may reform into thick bodies of "massive" or "excess" ice of drastically lower lithic contents than the underlying ground. In addition, processes such as thin-film transport (Sizemore et al., 2015) and thermal cycling (Fisher, 2005) can modify the state of the ice. Determining the vertical and horizontal structure of ice content will therefore inform us about the deposition and modification processes that have occurred over time. 
Recent efforts have focused on integrating multiple datasets and techniques to attempt to constrain aspects of the distribution and accessibility of mid-latitude ice. These include studies across swaths of the northern plains regions (Orgel et al., 2019; Ramsdale et al., 2019; Sejourne et al., 2019) as well as the nearly global (+/-60 latitude) Subsurface Water Ice Mapping (SWIM) Project (swim.psi.edu; Putzig et al., 2020). Overall, the scientific community has made significant progress in understanding the non-polar water ice on Mars, but further work is needed to decipher what this means for Mars' climate in the past, as well as the accessibility of the ice as an in situ resource for future human exploration. For example, how were the ice reservoirs emplaced? Which deposits reflect burial of snowfall versus those formed by vapor deposition? What processes affect snowfall and mantling, as well as subsequent modification, to the ice? How deep is the ice? What technologies would be needed to extract and utilize the ice in its current form?

We pose two overarching questions that should be a primary focus of Mars exploration during the next decade:

1. What climate record is preserved in mid-latitude ice deposits on Mars?

2. How accessible is the ice as a resource for future exploration?

We are reaching the limits of currently available datasets, just as we are starting to unlock the climate record and determine the three-dimensional extent of water resources contained within the Martian mid-latitudes. Ultimately, new missions are required to address the dearth of current data capable of resolving the upper tens of meters of the Martian subsurface within these dynamic mid-latitude regions and to supply ground truth for orbital discoveries.

\section{Critical Knowledge Gaps}

Although volatiles on Mars were already recognized as an important exploration target at the time of the last Decadal Survey, the major advances in subsurface water ice described above elevate its importance as a target for future exploration. In order to interpret the climate record in the mid-latitudes, as well as the accessibility for in situ resource utilization, several questions must be addressed regarding the ice-rich deposits:

1. What is the vertical structure and ice concentration? What are the emplacement and modification processes that generate and preserve this structure? How old are these deposits and how long did they take to form? How pervasive is layering and how many episodes of deposition/erosion are reflected in their stratigraphy?

2. What is the spatial distribution? Over what spatial scales are the thickness of the ice, as well as the depth to the top of the ice, heterogeneous?

3. What is the chemistry? What are the isotopic ratios, including $\mathrm{D} / \mathrm{H}$ ratios, in the ice? What impurities (e.g., dust, salts) are in the ice and in what concentrations? What climate signal(s) is/are preserved in the isotopic ratios and impurities in the ice, and what role do salts play in ice stability?

4. What is the near-surface atmospheric water vapor content at present day and how has it varied over previous orbital/axial cycles? Where is the ice in equilibrium with the current climate? And where is the ice out of equilibrium?

5. What is the relationship between observed geomorphic features and terrains and the amount and structure of subsurface ice at present day and in the past? 
6. What emplacement mechanisms and timeframes lead to massive (glacial) ice vs. pore-filling ice deposits? And what effect does this have on the properties (e.g., thickness, purity, distribution) of the resulting water ice reservoirs?

7. What are the characteristics of the ice-poor surface layer that insulates and protects non-polar ice deposits? What are the properties (e.g., thickness, structure, blockiness, porosity) of the debris layers over the various icy deposits?

8. What is the role of these deposits in creating habitable environments, either at present day or in the past?

\section{Approaches for the Next Decade}

Following up on recent discoveries, numerous studies by assessment groups and of mission concepts have taken place in the last five years to identify ways to address many of the questions listed above. These studies include reports from the MEPAG-organized Ice and Climate Evolution Science Analysis Group (ICE-SAG, 2019) and the Next Orbiter Science Analysis Group (NEX-SAG, 2015), results from a Keck Institute for Space Studies workshop (2017 KISS Report), and summary documents from the $6^{\text {th }}$ and $7^{\text {th }}$ International Conferences on Mars Polar Science and Exploration (Smith et al., 2018; Becerra et al., 2020), as well as from a 2018 Mars Workshop on Amazonian and PresentDay Climate (Diniega et al., 2018). Here, we highlight some of the key findings and techniques that are best poised to address the critical knowledge gaps outlined above.

- A need that has emerged and is almost ubiquitously recognized as one of the highest priorities by the Mars polar science and ice community, is that of a higher frequency orbital sounding radar to address the gap in our knowledge of the uppermost 10 meters of subsurface stratigraphy. Current radar assets at Mars include the MARSIS and SHARAD sounding radars, but these instruments were designed to probe relatively deep into the subsurface (hundreds of meters to kilometers). An L-band ( 1$2 \mathrm{GHz}$ ) or P-band ( 200-400 MHz) orbital radar would be able to resolve interfaces at finer spacing than the vertical resolution of current radar systems at Mars. Additionally, a ground-penetrating radar (e.g., RIMFAX onboard Mars 2020, the radar onboard China's Tianwen-1 mission, Zhou et al. 2016, or WISDOM onboard the ESA-Roscosmos ExoMars Rover mission, Ciarletti et al., 2017) with frequencies 50$400 \mathrm{MHz}$ onboard a rover sent to an icy mid-latitude target could resolve the structure of the upper tens of meters at that site. Clutter (reflections from non-nadir) can be confused with subsurface reflections and must be minimized with modeling from high-resolution terrain maps or multiple antennas and polarization techniques.

- Polarimetric synthetic-aperture radar (SAR) has never been flown at Mars. The polarization and strength of backscattered off-nadir radio waves are sensitive to the roughness of the surface and subsurface radar interfaces, as well as the dielectric, magnetic, and scattering properties of the shallow subsurface. L-P band wavelengths $(\geq 10 \mathrm{~s}$ of $\mathrm{cm})$ will be required to detect buried mid-latitude ice. SAR would be able to produce high-resolution ( $<50 \mathrm{~m} / \mathrm{pixel}) 2 \mathrm{D}$ images of the surface/shallow subsurface.

- Dielectric spectroscopy (a.k.a. impedance spectroscopy, spectral induced polarization, complex resistivity) is a diffusive surficial electromagnetic method where current is injected into the subsurface by galvanically coupled electrodes, capacitively coupled electrodes, or a magnetic dipole (loop). The corresponding voltage and phase are measured on a different pair of electrodes or separate loop, with separation 
distance determining the depth being investigated. The frequency of the injected current is varied over a large frequency range $(10 \mathrm{~Hz}-100 \mathrm{kHz})$, and the physical location of the electrodes or loops is then also varied. Many spectra are gathered and inversion techniques can be used to determine how the electrical properties change as a function of depth and frequency. The frequency response can be used to unambiguously detect ice (Stillman et al., 2010; Grimm and Stillman, 2015). Applications could involve multiple capacitive electrodes built into a "streamer" that is dragged behind a rover, a rover with loops in front and behind it, or multiple rovers.

- Thermal infrared imaging can be used to detect the depth and presence of subsurface ice by mapping seasonal temperature responses caused by ice's relatively high thermal inertia compared to that of regolith or dust (e.g., Bandfield, 2007; Piqueux et al., 2019). The next generation of thermal IR instruments (e.g., E-THEMIS; Christensen et al., 2017) represent a vast improvement ( 20 years of development) in resolution and precision compared to current instruments at Mars (e.g., THEMIS) and should allow for $\sim 50 \mathrm{~m} / \mathrm{pixel}$, multi-band (for simultaneous atmospheric measurements) thermal imaging to complement visible/VNIR datasets.

- Active source seismology (see Courville et al. 2020 WP) utilizes the role that surface/subsurface materials, including ice content, play on altering seismic velocity through a medium. Additionally, abrupt transitions in ice content, such as at the boundaries of an ice deposit, produce strong seismic-wave reflections and refractions. A roving system equipped with instruments to generate and record seismic vibrations (e.g., Courville et. al., 2018), or with passive-active acoustic seismic instrumentation (e.g., Lorenzo et al., 2019), could survey the depth and lateral extent of ice at the site.

- A high-resolution gravity field could enable mapping of shallow excess water ice deposits. A dedicated Martian geodesy mission would allow for geophysical analysis of the shallow and deep interior in unprecedented detail (see Sori et al. 2020 WP).

- Terrestrial analogue fieldwork will be crucial, not only to test future in situ resource excavation methods, but also to better understand glacial/periglacial processes and their relationship to climate, geomorphology, and ice content (see Grau Galofre et al. 2020 WP). In particular, polar deserts and regions of relict icy terrains provide a unique opportunity to connect field work, geomorphology, geophysics, and modeling efforts. In situ sampling and characterization systems being developed for Mars (e.g., Obbard et al. 2020) (see Edwards et al. 2020 WP) will benefit from testing at terrestrial analogue sites.

- Continued high-resolution imaging (see Dundas et al. 2020 WP) will be needed, as the discovery/detection (CTX-class imaging) and characterization (HiRISE-class imaging) of new impact craters which expose and excavate ice have been crucial for providing ground-truth of shallow massive ice in the mid-latitudes.

- An aerial rotorcraft (see Bapst et al. 2020 WP) or network of surface assets (see Diniega et al. 2020 WP) could acquire measurements of the distribution of water vapor in the boundary layer (5-10 km altitude) and the flux(es) of water vapor between the atmosphere and the surface, providing crucial constraints on models of ice evolution which are used to construct the history of Martian ice.

Overall, multiple methods can be used to detect ice. For example, SAR could produce a global map that can detect lateral heterogeneity of the top of the ice table and shed light into the ice's stability. A high-frequency sounding radar has the ability to detect lateral 
and vertical heterogeneities of the top and possibly bottom of ice as well as the ice's purity. A rover mission could then follow up on a site of interest, with GPR providing a much higher spatial resolution structural mapping at that location, and DS uniquely confirming the volume percent of ice laterally and vertically. Any of these landed techniques would be best applied by a mobile lander at a site with diverse ice-related geomorphology. This would enable characterization of the ice properties associated with many surface materials and landforms, improving interpretations across the planet.

Below are some recently developed mission architectures, concepts, and studies from the community that could be leveraged in the next decade to address the science above:

- COMPASS, a Discovery-class mission proposal that was submitted in 2019 for a new Mars orbiter to observe present climate processes and volatile transport contemporaneously with measurements of icy reservoirs (Byrne et al., 2020).

- MORIE, a Planetary Mission Concept Study (MORIE Final Report, 2020).

- MOSAIC, a Planetary Mission Concept Study (MOSAIC Final Report, 2020).

- Concepts developed for the ICE-SAG study, specifically "NF4", a stationary lander, and "NF5", an orbiter for global ice inventorying (ICE-SAG, 2019).

- GANGOTRI, an in situ mid-latitudinal concept with a retractable drill for englacial sampling up to hundred-meter depths (see Karunatillake et al., 2020 WP).

- Rover-based drilling to inspect for biosignatures (see Stoker et al., 2020 WP).

- Orbital mission designs developed within NEX-SAG (NEX-SAG, 2015).

- Mars Science Helicopter, an aerial platform for science investigations (see Bapst et al., 2020 WP).

Conclusion: Current datasets are unable to answer many of the outstanding questions that have emerged regarding Mars' mid-latitude ice. New missions will enable us to capitalize on the discoveries of the last decade and take the next giant leap forward in the next decade to address the questions posed above. A new orbital or landed mission focused on Martian ice would produce far-reaching scientific results, and would leverage the momentum and work being done in the community to identify approaches for addressing these questions.

\section{References}

Bandfield (2007), Nature, doi.org/10.1038/nature05781.

Becerra et al. (2020), Nature Astronomy, doi.org/10.1038/s41550-020-1127-y.

Bramson et al. (2015), GRL, doi.org/10.1002/2015GL064844.

Byrne et al. (2009), Science, doi.org/10.1126/science.1175307.

Byrne et al. (2020), $7^{\text {th }}$ Mars Polar Sci. Conf., Abstract \#6013.

Campbell \& Morgan (2018), GRL, doi.org/10.1002/2017GL075844.

Ciarletti et al. (2017), Astrobiology, doi.org/10.1089/ast.2016.1532.

Christensen et al. (2017), AGU Fall Meeting, Abstract \#P33H-01.

Courville et al. (2018), AGU Fall Meeting, Abstract \#P54D-02.

Diniega, Smith, \& Bramson (2018), Eos, doi.org/10.1029/2019EO114411.

Dundas et al. (2014), JGR-Planets, doi.org/10.1002/2013JE004482.

Dundas et al. (2018), Science, doi.org/10.1126/science.aao1619.

Fisher (2005), Icarus, doi.org/10.1016/j.icarus.2005.07.024.

Grimm \& Stillman (2015), Permafr. \& Periglac. Process. doi.org/10.1002/ppp.1833. 
Holt et al. (2008), Science, doi.org/10.1126/science.1164246.

Jakosky (1985), Space Sci. Rev., doi.org/10.1007/BF00241348.

Laskar et al. (2004), Icarus, doi.org/10.1016/j.icarus.2004.04.005.

Leighton \& Murray (1966), Science, doi.org/10.1126/science.153.3732.136.

Levy et al. (2014), JGR-Planets, doi.org/10.1002/2014JE004685.

Lorenzo et al. (2019), 50 th LPSC, Abstract \#3246.

Obbard et al. (2020), 7th Mars Polar Sci. Conf., Abstract \#6078.

Orgel et al. (2019), JGR-Planets, doi.org/10.1029/2018JE005664.

Pathare et al. (2018), Icarus, doi.org/10.1016/j.icarus.2017.09.031.

Piqueux et al. (2019), GRL, doi.org/10.1029/2019GL083947.

Plaut et al. (2009), GRL, doi.org/10.1029/2008GL036379.

Putzig et al. (2020), 51 st LPSC, Abstract \#2648.

Ramsdale et al. (2019), JGR-Planets, doi.org/10.1029/2018JE005663.

Séjourné et al. (2019), JGR-Planets, doi.org/10.1029/2018JE005665.

Sizemore, Zent, \& Rempel (2015), Icarus, doi.org/10.1016/j.icarus.2014.04.013.

Smith et al. (2009), Science, doi.org/10.1126/science.1172339.

Smith et al. (2018), Icarus, doi.org/10.1016/j.icarus.2017.06.027.

Squyres \& Carr (1986), Science, doi.org/10.1126/science.231.4735.249.

Stillman, Grimm, \& Dec (2010), J. Phys. Chem. B, doi.org/10.1021/jp9070778.

Stuurman et al. (2016), GRL, doi.org/10.1002/2016GL070138.

Toon et al. (1980), Icarus, doi.org/10.1016/0019-1035(80)90130-X.

Wilson et al. (2018), Icarus, doi.org/10.1016/j.icarus.2017.07.028.

Zhou et al. (2016), 16 ${ }^{\text {th }}$ Int. Conf. on GPR, doi.org/10.1109/ICGPR.2016.7572700.

\section{Reports:}

MEPAG ICE-SAG, 2019 Final Report

MEPAG NEX-SAG, 2015 Report

2017 Keck Institute for Space Studies Workshop, Final Report

MOSAIC 2019 Planetary Mission Concept Study, Presentation at MEPAG 38

MORIE 2019 Planetary Mission Concept Study, Presentation at MEPAG 38

\section{Other White Papers Submitted to this Decadal Survey:}

Bapst et al. (2020): "Mars Science Helicopter: Compelling science enabled by an aerial platform"

Courville et al. (2020): "Developing active source seismology for planetary science"

Diniega et al. (2020): "A Critical Gap: In situ Measurements of Planetary SurfaceAtmosphere Interactions Beyond Earth"

Dundas et al. (2020): "Current activity on the Martian surface: A key subject for future exploration"

Edwards et al. (2020): "Emerging capabilities for Mars exploration"

Karunatillake et al. (2020): "GANGOTRI mission concept on the glacial key to the Amazonian climate of Mars"

Grau Galofre et al. (2020): "A comparative view of glacial and periglacial landforms on Earth and Mars"

Sori et al. (2020): "Transformative science unlocked by future geodetic data at Mars, Venus, and Ocean Worlds"

Stoker et al. (2020): "We should search for extant life on Mars in this decade" 